\title{
ENTREVISTA AL DOCTOR CARLOS HuMBERTO REYES DÍA'Z
} ABRIL USCANGA BARRADAS

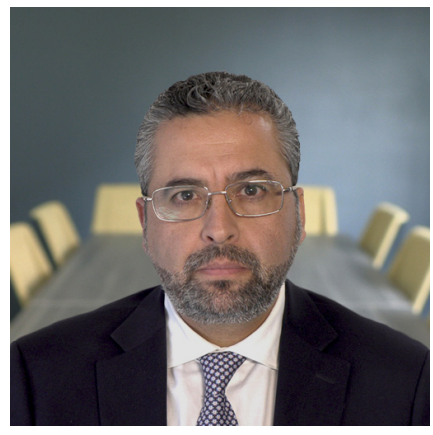

Carlos humberto Reyes díaz es Coordinador del Programa de Posgrado en Derecho, Profesor de Tiempo Completo en el Instituto de Investigaciones Jurídicas, Profesor de la Facultad de Derecho y miembro del Sistema Nacional de Investigadores del CONACYT.

\section{ENTREVISTA AL DOCTOR CARLOS HuMBERTO REYES DÍAZ}

Abril Uscanga Barradas: Buenos días, Doctor Carlos Humberto Reyes Díaz, muchas gracias por haber aceptado esta entrevista.

Carlos Humberto Reyes Díaz: Gracias a ustedes.

AUB: Doctor, quisiera iniciar la entrevista con unas cuestiones un poco más personales, permítame preguntarle, yo se que usted nació fuera de la Ciudad de México, ¿cuáles fueron sus motivaciones para estudiar Derecho en esta universidad?

${ }^{1}$ Este documento constituye una transcripción de la entrevista realizada. Se ha revisado sin alterar el estilo coloquial propio de una conversación, considerando que esta característica enriquecería el texto que se presenta al lector. La entrevista fue realizada con la colaboración de Diana Erika Hernández Ríos y Salvador Villaseñor Olguín. 
CHRD: Efectivamente, nací en la ciudad de Chihuahua, viví una época en la capital del Estado y después viví otros 7 años, aproximadamente, en la frontera, en Ciudad Juárez, en donde comencé mis estudios de la licenciatura en Derecho. Yo cursé en la Universidad Autónoma de Ciudad Juárez cinco semestres y al terminar estos 5 semestres, sentía que algo faltaba en mi vida. Además, para mí fue revelador el enterarme que el hijo de un amigo de mi papá se había ido a estudiar a la UNAM y que estaba pasando un momento genial, en esos momentos se me sembró algo en la cabeza y empecé a generar en mi mente la idea de que para mí sería lo ideal estudiar en la UNAM. Greo que para todos los estudiantes de provincia, la UNAM es un referente, quizá el máximo referente académico de México. Así empecé a hacer los tramites de un día para otro y, sin pensármelo dos veces, dije "si se abre la puerta, allá estaré; si no, culminaré la carrera en Ciudad Juárez”, por lo consiguiente, llegar a la UNAM fue para mi uno de esos grandes logros en mi vida.

AUB: Muy interesante, seguramente la historia de varios de los alumnos que hay en esta universidad. Doctor, podría comentarnos un poco de su experiencia ya estando aquí como alumno, ¿Quiénes fueron sus profesores, académicos o autores de libros que hayan marcado su trayectoria durante la carrera?

CHRD: Guando yo fui alumno de Derecho en Chihuahua, nosotros llevábamos los libros de profesores de la UNAM. Recuerdo que yo leía los libros y pensaba "algún día me gustaría conocer a estos autores de libros". En materia constitucional era irremediable tener las bases de Héctor Fix Zamudio, de Jorge Carpizo o de Ignacio Burgoa, pero fue hasta que llegue yo aquí que conocí a los grandes profesores de la Facultad de Derecho y del Instituto de Investigaciones Jurídicas, todavía vivos en aquella época, y desde luego me impactó conocer directamente a Eduardo García Máynez, a Ignacio Burgoa Orihuela, a Don Ernesto Gutiérrez y González, a Ignacio Galindo Garfias, a Cipriano Gómez Lara, en fin, una gran cantidad de profesores que para nosotros estaban en una plataforma inalcanzable, desde provincia. Verlos de cerca, platicar con ellos, tratarlos 
era, como estudiante, muy impactante y, sin embargo, a quien más recuerdo, no tanto por sus libros porque en realidad no escribió mucho, fue a mi tutor, paisano y amigo, Victor Carlos García Moreno, quien fue además mi director de tesis de licenciatura y quien creo yo, ha sido por mucho uno de los mejores profesores de Derecho Internacional de la Facultad de Derecho. Él murió hace más de 20 años, pero fue el que realmente me dejó las bases académicas más importantes de mi trayectoria. Si yo tengo una metodología de enseñanza es gracias a que trato de emular siempre cómo era él en el aula.

AUS: Le agradezco Doctor, por compartirnos esto, es usted uno de los muchos claros ejemplos de lo que significa que la universidad represente un proyecto nacional, sin embargo, en el interior de la república hay muchos jóvenes que aún no han tenido la oportunidad de trasladarse, ¿cuáles fueron los retos que usted afrontó durante la carrera y qué deudas tiene la universidad con los estados de la república?

GHRD: Quisiera partir esta respuesta en dos partes. Primeramente, sí, para mi fue difícil, actualmente hay muchos alumnos que quisieran estudiar en la UNAM y a mi parecer las condiciones cada vez son mejores, el que tiene ahora interés de estudiar en la universidad lo puede hacer con mucha más facilidad que antes. Cuando yo vine a estudiar a la UNAM, ni siquiera había internet, yo no sabía como comunicarme a la universidad excepto por una llamada telefónica, yo busqué un directorio telefónico de la Ciudad de México, encontré un teléfono de la UNAM y de ahí me fueron transfiriendo a otras líneas telefónicas hasta que encontré la correcta y, empecé a recibir la información que necesitaba. Hoy, con el simple hecho de tener acceso a internet, se puede ver claramente, ni siquiera se tiene que venir. Yo en ese entonces tuve que hacer todos los trámites y venir personalmente a hacerlos y regresarme a mi ciudad, después venir a presentar exámenes y regresar. Hubo ocasiones en las que en un mismo día llegaba a la Ciudad de México, hacia mi trámite y el mismo día en la noche regresaba en autobús, 24 horas de carretera no eran fáciles, pero yo lo disfrutaba porque leía todo lo que 
podía, tenía mucha ilusión de entrar a la UNAM. Y hoy en día, el estudiante que quiera de cualquier parte de la república, incluso del extranjero, tiene muchas más facilidades de acceder. Yo creo que es simplemente el tema de tomar la decisión, si yo pudiera decir en donde está la dificultad más grande para poder ingresar a la UNAM en este momento, viniendo de una entidad federativa diferente a la Ciudad de México, está en tomar la decisión de venir y cambiar su vida para estar en la universidad, aquí en México, es la decisión más difícil porque es separarse de la familia, quemar sus naves como dicen algunos. Y respecto a los retos a afrontar durante la carrera, para mí en principio fue la propia ciudad, el desconocimiento total de la ciudad fue un reto muy complicado, pero igual en gran medida me ayudó a conocer la universidad; yo sabía como trasladarme de mi casa a la universidad y de la universidad a mi casa en el transporte público, me sabía a la perfección el trayecto, pero de ahí en más no conocía, entonces para mí era llegar temprano a la universidad, porque además mis clases no eran continuas ya que siempre intentaba tener a los mejores profesores así que tomaba algunas clases muy temprano, a las 7:00am, otras a las 11:00am, otras por la tarde, otras por la noche, mi vida era estar todo el tiempo en la universidad, así que lo aproveché para recorrer todo el campus de Ciudad Universitaria, conocer todos los rincones posibles. Hoy me sorprendo, desafortunadamente, cuando le pregunto a mis alumnos si conocen un lugar en específico de la UNAM, ya estando ellos en último semestre, me dicen que no conocen la Biblioteca Nacional, el espacio escultórico, el jardín botánico, la zona cultural, etcétera. Me sorprende bastante ya que están a punto de dejar, como se los digo yo, la mejor etapa de sus vidas y no conocen lo que en su momento Fernando Serrano Migallón, uno de mis profesores y amigos más queridos, dijo: "la universidad es el secreto mejor guardado de México, hay que conocerla y vivirla, y la manera de vivirla es caminarla". En ese entonces representó un reto para mí no conocer la Ciudad de México, pero al mismo tiempo me abrió la posibilidad de conocer todos los rincones posibles de Ciudad Universitaria, esa es 
la razón por la que cada día tengo más ese sentido de pertenencia hacia la universidad.

AUB: Doctor, sabemos que usted ha es docente desde hace varios años. Ha llegado a consolidar su proyecto de vida dentro de la Universidad ¿Cuáles cree usted que son los obstáculos que actualmente representan el ámbito de la docencia y como se podrían superar?

GHRD: Respecto a la primera parte del planteamiento de la pregunta de la UNAM como mi proyecto de vida, es verdad, yo no tengo ningún interés de utilizar la academia como un espacio mientras encuentro otra oportunidad laboral, al contrario, cuando empecé como docente aquí en la Universidad supe que mi vida estaba vinculada irremediablemente a la UNAM y, no lo tomo como algo negativo e irremediable, sino como un destino al que cada día que me levanto lo afronto con mucho gusto en mi vida cotidiana, en ese sentido, yo quiero ser universitario toda mi vida. Por otra parte, los retos que considero más importantes para el docente, son dos principalmente: es indispensable estudiar todos los días, por mucho que uno imparta la misma asignatura cada semestre, cada momento de nuestra vida es diferente, es decir, del semestre en que yo imparto una materia al siguiente el mundo ya cambió, México cambió, es necesario mantenerse actualizado, ver cuales son todos los cambios académicos que uno puede incorporar en su estrategia docente y, por otro lado, hablando precisamente de esas estrategias docentes, creo yo que es cada vez más necesario establecer una relación profesor-alumno, y en eso reconozco que se encuentra uno de los retos mas importantes para mí, quiero lograr esa transición para que mis alumnos me vean no como el profesor de ese semestre, sino como el tutor de vida, tratando de imitar a esos profesores de los que yo guardo recuerdos cercanos, ellos eran profesores que no solamente me enseñaban en el aula, sino que me acompañaban y me daban una estrategia de como vivir la vida profesional. Este lo considero un reto muy importante, porque es un hilo muy delgado, se puede romper el hielo y al mismo tiempo se puede traspasar una barrera de confianza, de respeto, el cual también es indispensable 
tener en la relación con los alumnos. He sido docente por 18 años, y ahora estoy experimentando otra de las tareas importantes de la universidad, como es la investigación, vinculado ahora al Instituto de Investigaciones Jurídicas, no porque haya trascendido mi época docente sino porque la estoy complementando y esa, es la parte que me interesa, si yo quiero ser universitario de tiempo completo necesito conocer los espacios fundamentales de la tarea universitaria, que son la docencia, la investigación y la difusión del conocimiento.

AUB: Yo coincido con usted totalmente con respecto al reto primordial que tenemos los docentes, en particular con la relación profesor-alumno, que es una linea muy tenue, como lo ha comentado. Ahora bien, quisiera hacerle una pregunta de un tema que actualmente está incidiendo totalmente en nuestra universidad, que es tal vez uno de los más álgidos y que ha generado varias manifestaciones, el cierre de planteles. Me refiero al tema de las exigencias en materia de equidad de género. Desde su perspectiva, ¿cómo debe ser la relación alumno-profesor en la universidad, es decir, como regularla sanamente?

CHRD: Es una pregunta complicada, pero nuevamente la parto en dos. Las manifestaciones que hemos tenido en la universidad actualmente son manifestaciones legítimas. Lo que se está buscando es detener la violencia de género, el propio rector está tomando acciones encaminadas a solucionar este problema pero me parece que la vía por la cual se han reclamado estos derechos es la incorrecta. Yo le digo constantemente a mis alumnos que la mejor manera de que la universidad puede resolver sus problemas es discutiéndolos en un espacio abierto. Una universidad cerrada no contribuye a mejorar los problemas de la universidad, ni del país, ni en consecuencia los problemas de género. Las manifestaciones son perfectamente válidas y yo soy de los que cree que debe haber manifestaciones de inconformidad cuando se trata de defender este tipo de derechos, pero la universidad abierta es el espacio ideal para poder resolverlos, por ejemplo, las facultades cerradas hoy no son mejor de lo que eran antes de que cerraran sus puertas desde noviembre 
del año pasado; hoy los catedráticos valiosos que tiene la Facultad de Filosofía, entre otras, así como los estudiantes valiosos no están reunidos tratando de encontrar un problema a esto, están cada uno por su propio espacio o en grupos reducidos buscando la forma de cómo incidir para resolver el problema. Entonces, lo único que produce cerrar la universidad es quitarle al gobierno un contrapeso necesario para poder resolver los problemas nacionales, la universidad no puede dejar de ser ese espacio de diálogo. Respecto a la segunda parte de cómo debe ser la relación profesor-alumno, desafortunadamente las circunstancias impiden que volvamos a tener una relación como la que existía antes, es decir, yo escuchaba mucho cómo platicaban algunos profesores sobre una época en la que, por ejemplo, el Chato Noriega invitaba a sus alumnos a desayunar al Sanborns y ahí discutían y platicaban temas generales que no se podían ver en clase. Esa relación profesor-alumno ya no existe el día de hoy y si la existe, es mal vista, es más, hoy en día debemos tener incluso mucho cuidado de platicar con nuestros alumnos fuera del aula, porque eso nos pone en una circunstancia de sospecha. Ahora solo ven una posible situación de acoso por parte del profesor o de soborno por parte del alumno, es decir, todo se mira desde una perspectiva de sospecha. Desafortunadamente, hemos perdido esa confianza en la relación del maestro fuera de aula, el maestro referente, el maestro de vida, se ha perdido porque ha habido muchos abusos en ambas fronteras de la relación, ya sea del profesor o del alumno. ¿Y cómo hacerlo?, bueno, ahora hay que tratar de tener una relación respetuosa en la medida de lo posible, interactuar lo más institucional, académico, dentro de las instalaciones universitarias, hasta que por lo menos se eliminen o se creen protocolos mucho más efectivos para buscar que se erradiquen todas estas prácticas de violencia de género y violencia académica que evidenciamos día con día.

AUB: Gracias por su respuesta, ahora bien, pasando a otro tema ¿qué cree usted que les falta a los juristas de hoy en día?

CHRD: Yo estoy convencido de que cuando nosotros estudiamos Derecho nos enseñan que la norma jurídica es lo más importante 
para lograr la paz social y con el tiempo he podido entender que el Derecho es solamente una herramienta de transformación social, una herramienta de mejora continua de la sociedad, pero no es lo único que necesita un abogado, es decir, el abogado que cree que el derecho, así aisladamente, puede transformar a la sociedad tiene una visión muy limitada. Lo que necesita el jurista en la actualidad es conocer el Derecho, buscar que se aplique el Estado de Derecho, pero al mismo tiempo se tiene que nutrir de otras áreas del conocimiento para poder entender que nuestra sociedad es mucho más compleja, que no puede ser vista desde una sola óptica y que se necesita comprender el fenómeno social, la circunstancia política, la situación económica, el contexto internacional, la problemática del medio ambiente, en fin, muchos factores externos al ámbito jurídico y que, por otro lado, le ayudan al abogado a entender que la construcción de normas jurídicas se tiene que hacer una vez que conoces la sociedad en la que vives. Es absolutamente necesario tener cada vez un conocimiento más amplio, multidisciplinario y a partir de ese conocimiento, tratar de buscar, de tejer, la solución a los problemas por una vía interdisciplinaria.

AUB: Ahora quisiera pasar a un tema que está ligado con su labor alterna en esta universidad, me refiero a la labor que usted tiene fungiendo como coordinador del Programa de Posgrado en Derecho, conocemos que usted ha realizado esa labor por dos periodos, sin duda es un trabajo arduo que implica coordinar los esfuerzos de cuatro entidades: Acatlán, Aragón, el Instituto de Investigaciones Jurídicas y la Facultad de Derecho. Por favor, díganos desde su experiencia, ¿cuáles son los principios que deben regir un posgrado de calidad?

CHRD: Bueno, un posgrado de calidad se rige por principios que están establecidos, en primer lugar desde CONACyT, ya que dice cuales son las características que debe seguir un posgrado de calidad, pero yo quisiera salirme de esa óptica y hablar desde mi propia experiencia. Estoy a punto de cumplir dos periodos ahora en mayo de 2020 y, al terminar este encargo, lo que yo puedo decir es 
que, para empezar, la estructura de la universidad es una estructura bien diseñada, es un espacio muy generoso que permite tomar decisiones no de manera vertical, es decir, estar en el Posgrado me ha enseñado algo muy interesante de mi universidad: que las desiciones no se toman siempre por una sola persona, lo que hace o genera la gobernabilidad en la universidad es la decisión de los cuerpos colegiados. En el Programa de Posgrado en Derecho, aunque yo soy el coordinador, no soy el que toma las decisiones del Posgrado, estas son tomadas por el Comité Académico, es el órgano colegiado representado por las cuatro entidades que lo conforman, además de la representación de tutores y de alumnos, y la Coordinación. Pero esto sucede en toda la universidad, el rector toma sus decisiones que están en el mismo sentido del Consejo Universitario, la junta de gobierno que son los órganos más importantes, las propias facultades tienen su Consejo Técnico, los institutos se guían por los consejos, ya sea de humanidades o de investigación científica. Entonces, estar en el Posgrado de Derecho me ha ayudado a entender que la universidad toma decisiones de manera colegiada y eso siempre irá en beneficio de una mejora institucional. ¿Qué es lo que tenemos que hacer para poder hacer un mejor Posgrado? Justamente canalizar nuestras mejoras a partir de la toma de decisiones colegiadas. La gran idea que yo pueda tener para mejorar el Posgrado nunca será mejor que la que podamos discutir en el Comité Académico para poder hacer de este un Posgrado mejor. Entonces creo, sinceramente, que el Posgrado se fortalece en gran medida primero teniendo en la mira los objetivos de un Posgrado de calidad de CONAGyT, que tenemos que replantearnos todos los días qué es lo que queremos para un Posgrado y, además, un Posgrado no es ni tiene un objetivo único, se tiene un objetivo para el Maestro en Derecho y otro para el Maestro en Política Criminal, se tiene un objetivo para el Doctor en Derecho, como también se tiene y se debe tener un objetivo para los especialistas en algún área del Derecho. Entonces, cada finalidad se tiene que replantear todos los días, a dónde se quiere llegar y, con base en eso, CONAGyT nos ayuda a tener estrategias para 
poder definir la eficiencia terminal, la movilidad académica, el espacio de publicaciones, de difusión del conocimiento, etcétera. Pero más importantes que las líneas que plantea CONAGyT, es plantearnos todos los días hacia dónde queremos llegar como Posgrado, queremos ser el Posgrado de excelencia, pero no es solamente llegar sino mantenerse como tal y para eso necesitamos fortalecer nuestra planta de tutores, necesitamos fortalecer más las habilidades de conocimiento de nuestros alumnos, necesitamos fortalecer los procesos administrativos para hacer del Posgrado un espacio mucho más amigable, óptimo para el estudio de nuestros alumnos. Es decir, el que hace una maestría en Derecho tiene un objetivo que está muy vinculado a la docencia e iniciación a la investigación, el que hace doctorado tiene o debe de tener una mira mucho más enfocada a la investigación; necesitamos fortalecer esas competencias en los alumnos, necesitamos hacer que el alumno no piense solo en la obtención de un grado, sino en la adquisición de nuevas competencias para poder desarrollar su actividad profesional.

AUB: De acuerdo doctor, ahora me referiré específicamente a un punto del Posgrado, me voy a referir en este caso al doctorado avalado como un Posgrado de calidad a nivel internacional y que, sin duda, es un referente en el país. Quiero preguntarle al respecto, ¿cuál sería su recomendación para quienes desean realizar un doctorado? En particular, ¿qué nos pudiera decir, cuál es el objetivo de realizar un doctorado en Derecho en esta universidad?

CHRD: Cada vez que se habla del Posgrado, en la UNAM en general, y así lo han hecho saber mis colegas en las reuniones de coordinadores, se habla del Posgrado como la joya de la corona de la universidad, el grado máximo al que se puede acceder en nuestra universidad, la cúspide del conocimiento. Desafortunadamente, lo que yo he podido evidenciar, y creo que es en donde debemos trabajar en gran medida, es que el doctorado, al día de hoy por los que aspiran a él, lo ven como un título nobiliario, muchos de quienes quieren hacer un doctorado es porque aspiran a tener el título, quieren ponerse su toga, su muceta, su birrete con ínfulas, 
utilizar su venera, en fin, la forma, la parafernalia que representa, el glamour que rodea la investidura doctoral y por supuesto, además de eso, la apertura de puertas laborales, etcétera. Yo creo que esos son elementos adicionales interesantes, pero no son la esencia del doctorado, ya que este es una competencia que se adquiere por la cual uno se puede ostentar como mejor investigador de un área del conocimiento. Quien hace un doctorado en Derecho no tiene que perder de vista que la competencia que va a adquirir es ser un mejor investigador del hecho social y del derecho como una herramienta de mejora. El hecho de ser doctor no implica ser mejor abogado, no se es mejor profesor, simplemente se adquieren algunas herramientas para ser investigador. Ahora, por supuesto que sirve porque en nuestra realidad actual, los problemas nacionales e internacionales tienen que ser analizados por quienes investigan realmente el problema de fondo, así que quien quiera ser doctor en Derecho tiene que reconocer que en una investigación profunda, seria, rigurosa, completa, se va a encontrar o se puede llegar a encontrar una hipótesis que puede ser, o no, la solución a un problema nacional o internacional. Entonces, quien quiera ser doctor debería tener en cuenta que está adquiriendo una competencia que lo hace responsable para ser un investigador riguroso de los problemas y pueda darle a conocer a los gobiernos, a los profesores y alumnos, a la comunidad en general, cuáles son las alternativas posibles para solucionar algunos problemas.

AUB: Ahora, Doctor, quiero preguntarle con relación al mismo Posgrado y la experiencia que usted ha tenido en él. ¿Cuáles considera usted que son las fortalezas actuales del Posgrado y cuáles son las áreas de oportunidad que deben de ser tratadas para la siguiente gestión?

GHRD: En lo personal, no quiero dejarle a quien me suceda en el Posgrado alguna tarea, ni decirle cual es el camino que tiene que seguir, ya que cada universitario tiene una visión diferente. Por supuesto, cuando uno llega a la Coordinación del Posgrado se enfrenta con nuevos problemas, con realidades distintas a las que quedaron 
en la administración anterior y lo único que sí puedo decir es que nuestro Posgrado actualmente es fuerte, uno de los más fuertes del país y, me atrevo a decir, es más fuerte que incluso muchos Posgrados extranjeros ya que tenemos una planta de tutores muy relevante, cerca de 600 tutores de los cuales un porcentaje importante pertenecen al Sistema Nacional de Investigadores, son investigadores nacionales de calidad, son tutores que publican, tutores que enseñan, tutores que incluso imparten cursos en las entidades federativas a otros profesores, a gente de la administración pública, a gente del Poder Judicial, personas de la iniciativa privada, es decir, tenemos una planta de tutores que ninguna otra institución en el país tiene, en el campo del Derecho. Tenemos alumnos que tienen un alto nivel de pertenencia a nuestra universidad; el aspirante que llega a ser alumno en la universidad, incluso a nivel posgrado, automáticamente se siente universitario para toda la vida, como lo decía en su momento el rector José Narro: "entrar a ella es difícil, salir es imposible". Ser universitario y en el máximo nivel que otorga la universidad, el posgrado, genera una pertenencia y un sentido de responsabilidad social que no se genera en otros posgrados, que generalmente tratan de adquirir competencias laborales, competencias profesionales, aquí en la universidad, además, se trata de transmitir un tema de responsabilidad social, de compromiso social e institucional con México y con nuestra universidad, entonces esa es una de nuestras fortalezas principales. Por supuesto, hay que destacar todo el ámbito académico, tenemos espacios muy importantes de movilidad, damos a nuestros estudiantes la posibilidad de movilidad nacional e internacional que a mí no me tocó cuando era estudiante, sí había posibilidades, pero mucho más reducidas; al día de hoy nuestro Posgrado, por el hecho de estar en el PNPG de CONACyT, tiene muchas puertas presupuestales para poder acceder a espacios de movilidad en otras universidades, experimentar la realidad extranjera que es un elemento indispensable para reconocer no a México como el centro del mundo, sino como un espacio más que se tiene que comparar con otras partes del mundo para saber como resolver los 
problemas que nosotros experimentamos, ya que no son exclusivos de México; tenemos que ver qué es lo que se hace en otras partes del mundo para evitar inventar la "receta mexicana", sino tratar de aspirar a llegar a soluciones mucho más globales. Yo siempre he pensado que los problemas globales no se pueden solucionar desde una perspectiva nacional, por lo tanto la experiencia, la exposición extranjera de nuestros alumnos, les ayuda a entender un mundo más complejo del que representa nuestra sociedad nacional y eso lo tenemos aquí. Nuestros alumnos, un porcentaje muy importante de ellos se va, tenemos un porcentaje aproximadamente del $50 \%$ de nuestros alumnos que gozan de una beca de estudios que les permite tener ingresos y, precisamente, no abandonar sus estudios por falta de recursos económicos. Entonces sí estamos en una posición privilegiada como Posgrado y, precisamente por ello, tenemos que fortalecer esta posición. ¿Qué le podría yo decir del reto más importante del Posgrado a mi sucesor? Necesariamente mantener nuestra calidad en el Posgrado desde la evaluación del CONACyT. Mi sucesor o sucesora tendrá que enfrentar esta evaluación, que es muy compleja, pero que nos ayuda al mismo tiempo a hacer una mirada introspectiva para saber en dónde estamos fallando y hacia dónde tenemos que caminar para mejorar nuestro Posgrado. Tenemos que propiciar mayores publicaciones, especialmente derivadas de la relación tutor/alumno, tenemos que seguir incidiendo en los sectores público, privado y social, es necesario fortalecer la competencia de que nuestros tutores y alumnos escriban y expongan en foros internacionales, para ello se requiere dominio del inglés.

AUB: Muchas gracias Coordinador, por estas recomendaciones y este aprendizaje en relación con el Posgrado que usted conoce tan bien. Quisiera ahora entrar en una de las preguntas finales, queremos ahondar un poco en su trabajo como investigador, quisiéramos saber un poco acerca de sus líneas de investigación y si nos pudiera comentar un poco acerca de ellas, así como indicarnos uno de los libros que tendrían que leer quienes estén adentrándose a su linea de investigación, a su área del conocimiento. 
CHRD: Yo he trabajado en temas de comercio internacional, prácticamente 18 años en la docencia, actualmente en el Instituto de Investigaciones Jurídicas tengo a mi cargo el área de Derecho Internacional Privado y especialmente el tema de inversión extranjera. El Derecho Internacional Privado lo comparto con otra investigadora del Instituto, el tema de comercio internacional es compartido con otros colegas del propio instituto, pero si yo pudiera hablar de mi linea de investigación en concreto, diría que está directamente vinculada con la protección internacional de inversiones. El hecho de que los gobiernos tengan libertad absoluta para tomar decisiones gubernamentales que pueden llegar a afectar derechos de propiedad o derechos de inversión, son riesgos latentes de posibles demandas internacionales. Yo tengo esa linea de investigación para tratar de encontrar de qué manera los gobiernos pueden tomar decisiones de forma mucho más eficientes, quizá con base en estudios científicos y técnicos mucho más precisos para que las decisiones gubernamentales no sean simples actos de autoridad, sino que sean actos de autoridad con base científica. Esto elimina muchos riesgos para posibles demandas internacionales, que al día de hoy están creciendo mucho. México ha sido demandado ya en varias ocasiones por empresas, por inversionistas extranjeros, debido a decisiones que se han tomado en el camino de manera incorrecta, México también ha ganado controversias, pero en las que hemos perdido se ha tenido que pagar indemnizaciones muy caras que forman parte del presupuesto, de nuestros ingresos nacionales, son recursos que a su vez representan nuevas posibilidades para generaciones futuras, entonces el hecho de tomar una decisión incorrecta por parte del gobierno implica la posibilidad de una demanda que nos puede llevar al pago de indemnizaciones altísimas. He decidido tener esta linea de investigación para tratar de sembrar en el gobierno la necesidad de que las decisiones gubernamentales tienen que ser cada vez más congruentes, más pulcras jurídica y técnicamente, con mira a la construcción de políticas públicas que hagan sentido y no que sean solamente caprichos del gobierno en turno. Con base en eso, creo 
yo, que ahí es donde se centra fundamentalmente mi línea de investigación y que, por supuesto, en mi etapa como miembro del Instituto de Investigaciones Jurídicas trataré de fortalecer a partir de las investigaciones que mis colegas realizan para poder hacer de esta propuesta algo multidisciplinario y encontrar diferentes maneras de ver el problema. En cuanto a la recomendación de algún libro, es una pregunta también muy complicada porque yo siempre le digo a mis estudiantes que los libros son una versión que tenemos los autores de determinados hechos, yo les recomiendo a mis alumnos que utilicen las fuentes primarias, que no necesariamente son libros. Si alguien quiere aprender de estos temas de inversión siempre va a haber algún autor nacional o extranjero que pueda opinar sobre esto, pero creo que lo que más ayuda a entender esta problemática son las decisiones de los Tribunales Internacionales que reflejan claramente cuáles son las fallas que tienen los gobiernos al momento de adoptar sus políticas nacionales. Sí hay libros y en esto tengo que admitir que cuando yo construyo mi conocimiento en la materia, lo hago tomando como base siempre a autores extranjeros. Hay algunos autores nacionales que han tratado estos temas, yo mismo he tenido algunas aproximaciones a los temas, pero me parece que la principal fuente de conocimiento de esta materia es en inglés. En alguna ocasión, cuando tuve la oportunidad de hacer una estancia breve de investigación en el Instituto Max Planck de Derecho Internacional Público y Comparado de Heidelberg, Alemania, algún colega mío me decía "Todo lo que escribo en materia jurídica lo hago en alemán", él era originario de Alemania, "pero si yo quiero que el mundo me lea, debo escribir en inglés". El inglés, al día de hoy, es el idioma más importante para la transmisión del conocimiento jurídico, así que creo yo que hay una cantidad tremenda de obras en materia de protección de inversiones que están en inglés y sí, por supuesto, ahí hay autores como Foost Pauwelyn o Zachary Douglas, quienes son algunos referentes importantes de los teóricos actuales en materia de protección de inversiones, pero insisto en que la fuente principal del conocimiento en estos temas debe ser la 
fuente original, las decisiones de los Tribunales Internacionales, los propios tratados de inversión, leyes nacionales. A partir de ahí, el que está interesado en estos temas puede crear su propia versión y, por supuesto, hay que enriquecerla con los teóricos internacionales, pero siempre es importante partir de la fuente primaria.

AUB: Finalmente, quisiera hacerle una última pregunta, no quiero abusar mucho de su tiempo. Quisiera saber Doctor, ¿qué representa para usted la UNAM?

CHRD: Para mí la universidad representa varias cosas: representa un símbolo nacional, creo que la universidad es uno de esos símbolos más importantes que tenemos, la UNAM es una habitación más de mi casa, la UNAM es o lo fue en alguna época el objetivo que cumplí, pero al mismo tiempo cada día representa mi compromiso profesional más importante, si yo me levanto todos los días a realizar mi tarea es porque sé que vengo a la UNAM y quiero que esta institución siga siendo el referente más importante de nuestro país en materia jurídica. Se ha dicho, y quizá se ha abusado en algunas ocasiones de decir, que la universidad es el referente, la conciencia crítica de la nación y a veces pienso que esas palabras de tanto que se repiten se dan por sentadas y me parece que ahí tenemos que trabajar mucho. La universidad precisamente tiene que luchar para seguir siendo la conciencia crítica de la nación, pero para serlo es necesario hacer cada día de ella un espacio mejor para trabajar, por ello insisto, la universidad cerrada no puede ser la conciencia crítica del país. Greo que la universidad es hoy por hoy una de las instituciones más generosas que tiene México y tenemos que protegerla. Es todo eso y más, hablar de la universidad siempre hace que la respuesta no salga de la mente, sino del corazón, siempre que me expreso de mi universidad siento que estuviera hablando de mi propia hija, de mi madre, siento que me llena de orgullo ser parte de una institución como esta. Me alegra mucho platicar de ella, pero al mismo tiempo me vuelve vulnerable como persona saber cuando está amenazada, cuando está en riesgo, me hace pensar que esa gran fortaleza que tiene como institución en 
nuestro país al mismo tiempo se puede ver vulnerada en espacios cerrados. La llevo en el corazón, es la institución más importante en mi vida, pero al mismo tiempo la quiero como si fuera alguien de mi familia y precisamente por ello temo igual que temo por la vida o salud de mi familia. Formo parte de esta institución con mucho orgullo y quiero hacer de ella el espacio más importante de México trabajando todos los días para ello.

AUB: Le agradezco muchísimo Doctor, Coordinador, en verdad que nos sentimos muy complacidos de tener esta entrevista, le agradecemos profundamente no solamente por esta ocasión si no por todas las veces que hemos tenido la oportunidad de convivir con usted. Su periodo, como usted lo ha comentado, está a punto de concluir, pero queremos hacerle patente por parte de la Revista que ha sido un pilar para la creación de conocimiento y, nos sentimos muy afortunados de haber convivido con usted en estos 6 años que se van a cumplir. Le expresamos un profundo agradecimiento por la confianza y las oportunidades que ha brindado a esta Revista.

GHRD: El trabajo que se está haciendo en esta revista va subiendo en su calidad gracias a lo que se ha hecho con ella en los últimos años, que ha sido gracias a las aportaciones que ha tenido nuestra comunidad y de quien ha organizado los esfuerzos de cada publicación.

AUB: Le agradecemos nuevamente Doctor, muchísimas gracias. Le deseamos mucho éxito en sus siguientes proyectos. 
